\title{
Colchicine attenuates renal fibrosis in a murine unilateral ureteral obstruction model
}

\author{
SEIJI ITANO, MINORU SATOH, HIROYUKI KADOYA, YUJI SOGAWA, \\ ATSUSHI UCHIDA, TAMAKI SASAKI and NAOKI KASHIHARA \\ Department of Nephrology and Hypertension, Kawasaki Medical School, Kurashiki, Okayama 701-0192, Japan
}

Received April 11, 2016; Accepted February 23, 2017

DOI: $10.3892 / \mathrm{mmr} .2017 .6539$

\begin{abstract}
The present study aimed to assess the effects of colchicine, a known anti-inflammatory agent, on renal fibrosis using a murine model of unilateral ureteral obstruction (UUO). Male C57BL/6 mice were divided into two groups, vehicle- and colchicine-treated. Colchicine $(0.5 \mathrm{mg} / \mathrm{kg} / \mathrm{day})$ was administered by osmotic pump, and the UUO procedure was performed on the left kidney 7 days later. The mice were sacrificed at 14 days following UUO. Colchicine treatment suppressed interstitial fibrosis of the UUO kidneys. In addition, fibrogenic gene expression in the UUO kidneys was decreased by colchicine administration. NRK-49F normal rat kidney fibroblasts were cultured with or without colchicine under angiotensin II stimulation, following which a wound-healing assay and actin fiber staining were performed to evaluate the effects of colchicine in vitro. Colchicine was demonstrated to inhibit angiotensin II-induced fibroblast migration in vitro in a concentration-dependent manner. Colchicine treatment also suppressed the angiotensin II-induced activation of Ras homolog gene family member A in NRK-49F cells. In conclusion, colchicine treatment significantly inhibited fibroblast activity in vitro and attenuated renal fibrosis in vivo in UUO-operated mice. Therefore, the prevention of renal fibrosis following injury may represent a novel therapeutic application for colchicine.
\end{abstract}

Correspondence to: Dr Minoru Satoh, Department of Nephrology and Hypertension, Kawasaki Medical School, 577 Matsushima, Kurashiki, Okayama 701-0192, Japan

E-mail: msatoh@med.kawasaki-m.ac.jp

Abbreviations: CKD, chronic kidney disease; UUO, unilateral ureteral obstruction; $\alpha$-SMA, $\alpha$-smooth muscle actin; LDH, lactate dehydrogenase; RhoA, Ras homolog gene family member A; EMT, epithelial-mesenchymal transition

Key words: colchicine, drug repositioning, fibrosis, kidney, myofibroblast, Ras homolog gene family member A, ureteral obstruction

\section{Introduction}

Tubulointerstitial fibrosis is a final common pathway that leads to chronic kidney disease (CKD) progression (1). Renal fibrosis is associated with the prognosis of several kidney diseases, and suppression of fibrosis progression may protect renal function. Renal interstitial fibrosis is characterized by inflammatory cell infiltration, interstitial fibroblast proliferation, tubular atrophy and excessive accumulation of extracellular matrix (2). Several drugs currently used for other disorders may also exert antifibrotic activity. Blockers of the renin-angiotensin-aldosterone system and inhibitors of 3-hydroxy-3-methyl-glutaryl coenzyme A reductase have been demonstrated to prevent fibrosis in cardiovascular and renal diseases (3). While specific targeting of drugs to kidney cells would be desirable, this technology only exists at the preclinical stage and with limited success (4). Therefore, drug repositioning, i.e., reinvestigation of existing drugs for novel therapeutic indications, has been a trending topic in drug development (5).

Colchicine has been traditionally used for the prevention and treatment of gout and acute gout, respectively (6). The drug is known to exert anti-inflammatory action by inhibiting migration and chemotaxis of neutrophils and other motile cells via inhibition of microtubule polymerization (7). Colchicine is also used to treat Behçet's syndrome, primary biliary cirrhosis and familial Mediterranean fever (8). It is also beneficial for the treatment or prevention of cardiovascular diseases, including pericarditis, postsurgical atrial fibrillation and acute cardiovascular syndromes (8). Furthermore, colchicine has been demonstrated to possess antifibrotic properties mediated by suppressing collagen synthesis and secretion in hepatic and pulmonary fibrosis experimental models $(9,10)$. Previous studies have demonstrated that colchicine prevents renal injury in animal models of chronic cyclosporine nephrotoxicity (11), anti-glomerular basement membrane glomerulonephritis (12), diabetic nephropathy (13) and hypertensive CKD (14). Therefore, previous evidence suggests that colchicine may serve as a repositioned drug for the treatment of kidney diseases.

Unilateral ureteral obstruction (UUO) is a well-established experimental model of renal tubulointerstitial fibrosis (15). Several factors are important in the process of UUO-mediated fibrosis, including macrophage inflammation (16), pre-inflammatory cytokine/chemokine production (17), 
angiotensin II (18) and fibroblast activation (19). The present study aimed to investigate whether colchicine attenuates tubulointerstitial fibrosis in a mouse model of UUO, and whether colchicine has potential as a novel repositioned drug for the treatment of renal fibrosis.

\section{Materials and methods}

Animals. Male C57BL/6 mice were purchased from the Central Laboratory for Experimental Animals, Inc. (Osaka, Japan) and housed in a temperature $\left(22 \pm 2^{\circ} \mathrm{C}\right)$ and humidity $(40 \pm 20 \%)$-controlled room under a $12 \mathrm{~h}$ light-dark cycle. The mice were given free access to tap water and standard laboratory chow (MF; Oriental Yeast Co., Ltd., Tokyo, Japan). All animals were cared for in accordance with the guidelines set forth by the Guide for the Care and Use of Laboratory Animals 8th Edition (National Institutes of Health, Bethesda, MD, USA), and the present study was approved by the ethics committee of the Kawasaki Medical School (Kurashiki, Japan; approval no. 13-051).

Experimental groups and protocol. Male mice (age, 8 weeks; weight, 23-28 g) were randomly divided into two groups, vehicle-treated $(n=8)$ and colchicine-treated $(n=14)$. Colchicine (0.5 mg/kg/day; cat. no. C9754; Sigma-Aldrich; Merck KGaA, Darmstadt, Germany) was diluted into saline and administered by ALZET $^{\circledR}$ osmotic pumps (model no. 1004; Durect Corporation, Cupertino, CA, USA) over 3 weeks, which were implanted under the skin of the dorsal region. The UUO procedure was conducted 7 days following the pump insertion, by ligation of the left ureter as previously described (18). The right, non-UUO-operated kidneys were used as control samples. A total of 14 days following UUO, the body weight was recorded, the mice were sacrificed, and their kidneys were carefully extracted and weighed. Blood samples were obtained at the time of death for measurement of serum creatinine and serum alanine aminotransferase. The levels of alanine aminotransferase and creatinine were measured by a commercial laboratory at SRL, Inc. (Tokyo, Japan) using an automatic inspection device (DRI-CHEM, FDC-700; Fujifilm Corporation, Tokyo, Japan).

Histology and immunohistochemistry. Kidney tissues were fixed in $4 \%$ paraformaldehyde overnight at room temperature. Then, tissues were dehydrated in graded alcohols and embedded in paraffin using standard techniques. Kidney sections ( $4 \mu \mathrm{m}$ thick) were prepared from paraffin-embedded tissues. The sections were de-waxed, re-hydrated by standard techniques and stained with Masson's trichrome stain. Images were then captured using a BZ-9000 fluorescence microscope (Keyence Corporation, Osaka, Japan). For immunohistochemistry, deparaffinized serial sections were rehydrated in PBS and subjected to antigen retrieval by microwave heating (500 W, $15 \mathrm{~min})$. Sections were washed with PBS and incubated in $0.3 \%$ hydrogen peroxide (in $1 \mathrm{X}$ PBS) to block endogenous peroxidase activity, prior to further incubation with the primary antibodies for $1 \mathrm{~h}$ at $37^{\circ} \mathrm{C}$ in a humidified chamber. Primary antibodies against vimentin (dilution, 1:500; rabbit polyclonal; cat. no. sc-7557R; Santa Cruz Biotechnology, Inc., Dallas, TX, USA) and $\alpha$-smooth muscle actin ( $\alpha$-SMA; dilution, 1:100; rabbit polyclonal; cat. no. ab5694; Abcam, Cambridge, UK) were used and detected using the Histofine Simple Stain MAX-PO kit (Nichirei Corporation, Tokyo, Japan) and 3,3'-diaminobenzidine (Sigma-Aldrich; Merck $\mathrm{KGaA}$ ). The severity of tubulointerstitial injury was evaluated by examining 10 optical fields in randomly selected tissue samples. Images of scarred areas (stained blue with Masson's trichrome) and areas with positive staining for vimentin and $\alpha$-SMA (brown) were quantified using a color image analyzer (Win ROOF version 5.6; Mitani Co., Fukui-city, Fukui, Japan). Glomeruli, tubules and blood vessels of the cortex were excluded. Results were presented as a $\%$ of the relative volume of the scanned interstitium.

Reverse transcription-quantitative polymerase chain reaction (RT-qPCR). Total RNA extraction and RT-qPCR were performed as described previously (20). Briefly, total RNA was isolated from kidneys using TRIzol ${ }^{\circledR}$ (Invitrogen; Thermo Fisher Scientific, Inc., Waltham, MA, USA), followed by digestion with DNase (Sigma-Aldrich; Merck KGaA). cDNA was synthesized from total RNA $(1 \mu \mathrm{g})$ using Moloney murine leukemia virus reverse transcriptase (Thermo Fisher Scientific, Inc.) with oligo $(\mathrm{dT})_{12-18}$ as a primer (Thermo Fisher Scientific, Inc.). Reverse transcription was performed for $50 \mathrm{~min}$ at $37^{\circ} \mathrm{C}$ according to the manufacturer's protocol (Thermo Fisher Scientific, Inc.). Primers and probes for TaqMan analysis were designed using sequence information from GenBank (National Institutes of Health) (21) and the Primer3 online software (http://frodo.wi.mit.edu/primer3/; accessed July 1, 2015). Primer and probe sequences are listed in Table I. TaKaRa Premix Ex Taq (Takara Bio, Inc., Otsu, Japan), with a final reaction volume of $20 \mathrm{ml}$, was used for the TaqMan probe-based RT-PCR reaction, which was performed on a Applied Biosystems 7500 Fast Real-Time PCR System (Applied Biosystems; Thermo Fisher Scientific, Inc.) with the following cycling conditions: $2 \mathrm{~min}$ at $50^{\circ} \mathrm{C}$, $10 \mathrm{~min}$ at $95^{\circ} \mathrm{C}, 55$ cycles of $15 \mathrm{sec}$ at $95^{\circ} \mathrm{C}$ and $1 \mathrm{~min}$ at $60^{\circ} \mathrm{C}$. The level of mRNA expression in each sample was quantified using the absolute quantification standard curve method (22). Plasmid cDNA of each gene was used to prepare absolute standards. The concentration was measured using the $\mathrm{A}_{260}$, which was converted to the number of copies using the molecular weight of the DNA. Each mRNA expression levels were normalized to those of the housekeeping $18 \mathrm{~S}$ ribosomal RNA gene.

Cell culture. NRK-49F cells, a fibroblastic clone of normal rat kidney cells, were purchased from the Japanese Collection of Research Bioresources Cell Bank (Osaka, Japan), The cells were cultured in Dulbecco's modified Eagle's medium supplemented with $5 \%$ fetal bovine serum (FBS), $1 \%$ non-essential amino acids and 1\% penicillin/streptomycin (all Sigma-Aldrich; Merck KGaA). Confluent cells at passages 4-10 were used in subsequent experiments.

Cell viability and lactate dehydrogenase (LDH) assays. Effects of colchicine on cell viability were evaluated using the Premix (2-(4-Iodophenyl)-3-(4-nitrophenyl)-5-(2,4disulfophenyl)-2H-tetrazolium sodium salt (WST-1) Cell 
Table I. Sequences of primers and probes used for quantitative polymerase chain reaction.

\begin{tabular}{|c|c|c|}
\hline Gene & Accession number & Primer and TaqMan probe sequences $\left(5^{\prime}-3^{\prime}\right)$ \\
\hline Fibronectin & NM_010233 & $\begin{array}{l}\text { Forward primer: ATGATGAGGTGCACGTGTGT } \\
\text { Reverse primer: TGACGCTTGTGGAACGTGT } \\
\text { TaqMan probe: FAM-TCGTGGAGAATGGGCATGCA-TAMRA }\end{array}$ \\
\hline$\alpha 1$ type I collagen & NM_007742 & $\begin{array}{l}\text { Forward primer: TGTGCGATGACGTGCAAT } \\
\text { Reverse primer: TTGGGTCCCTCGACTCCTAC } \\
\text { TaqMan probe: FAM-ACTGGACTGTCCCAACCCCCAAAG-TAMRA }\end{array}$ \\
\hline E-cadherin & NM_010217 & $\begin{array}{l}\text { Forward primer: CGTGTACCCAGGTCTCAGAAG } \\
\text { Reverse primer: TTGTTTCTTTGTCCCTGTTGG } \\
\text { TaqMan probe: FAM-ACGAGACTGGGTCATCCCTCCCAT-TAMRA }\end{array}$ \\
\hline $18 \mathrm{~S}$ rRNA & NR_003278 & $\begin{array}{l}\text { Forward primer: CCTGCGGCTTAATTTGACTC } \\
\text { Reverse primer: GACAAATCGCTCCACCAACT } \\
\text { TaqMan probe: FAM- TCTTTCTCGATTCCGTGGGTGGTG -TAMRA }\end{array}$ \\
\hline
\end{tabular}

FAM, 6-carboxyfluorescein; TAMRA, N,N,N',N'-tetramethyl-6-carboxyrhodamine derivative.

Proliferation Assay system (cat. no. MK400; Takara Bio, Inc.). LDH levels (an indicator of cell injury) were measured using the LDH Cytotoxicity Detection kit (cat. no. MK401; Takara Bio, Inc.). These assays were conducted according to the manufacturer's protocols. Cells ( $1 \times 10^{3}$ cells) were seeded in 96-well plates and were co-incubated with $100 \mathrm{nM}$ angiotensin II (cat. no. A9525; Sigma-Aldrich; Merck KGaA) and 0-10 nM colchicine (cat. no. C9754; Sigma-Aldrich; Merck KGaA) for 24 h, prior to performing the WST-1 or LDH assays.

Ras homolog gene family member A (RhoA) activity assay. RhoA activity was assessed using the RhoA G-LISA Activation Assay Biochem kit (cat. no. BK121; Cytoskeleton, Inc., Denver, CO, USA) according to the manufacturer's protocol. Cells were incubated with 0 to $10 \mathrm{nM}$ colchicine at $37^{\circ} \mathrm{C}$ for $30 \mathrm{~min}$, followed by treatment with $100 \mathrm{nM}$ angiotensin II. The cells were collected for analysis $12 \mathrm{~min}$ post-stimulation. Cells were lysed with lysis buffer containing Tris $\mathrm{pH} 7.5, \mathrm{MgCl}_{2}, \mathrm{NaCl}$, nonionic detergent and SDS. Cell lysates were then incubated in a Rho-GTP affinity plate with binding buffer for $30 \mathrm{~min}$ at $4^{\circ} \mathrm{C}$ prior to further incubation with the anti-RhoA primary antibody for $45 \mathrm{~min}$ at room temperature. Following plate washing with kit wash buffer, the appropriate secondary antibody was added and incubated at room temperature for $45 \mathrm{~min}$. Following incubation, the plate was washed with kit wash buffer again then the mixed horseradish peroxidase detection reagent was added into each well. The luminescence signals were detected using a microplate luminescence reader (23). All antibodies and reagents used for the RhoA activity assay were provided by the Cytoskeleton, Inc. kit.

Wound-healing assay. Cells were grown to confluence in $10 \mathrm{~cm}$ plates, and a sterile $200 \mu \mathrm{l}$ pipette tip was used to scratch the cell layer. The cells were washed twice with PBS, after which medium supplemented with $100 \mathrm{nM}$ angiotensin II, 0.5\% FBS and 0-10 nM colchicine was added. An inverted microscope (IX81N; Olympus Corporation, Tokyo, Japan) was used to assess the cells at 0 and $20 \mathrm{~h}$. Cell migration rates (\%) were calculated using the following formula: [ $(0 \mathrm{~h}$ scratch area-20 $\mathrm{h}$ scratch area)/0 h scratch area]x100.

Actin fiber staining. Actin fibers were stained with Acti-stain 555 phalloidin (cat. no. PHDH1-A; Cytoskeleton, Inc.). Fibroblasts were cultured as aforementioned prior to the wound-healing assay, and actin staining was performed according to the manufacturer's protocol. Briefly, the culture medium was removed, and the cells were gently washed with PBS prior to fixation with paraformaldehyde (3.7\%) for $10 \mathrm{~min}$. The cells were washed again with PBS, permeabilized with $0.5 \%$ Triton X-100 for $5 \mathrm{~min}$, washed, and stained with phalloidin for $30 \mathrm{~min}$ in the dark at room temperature. Nuclei were stained with Hoechst 33342 (cat. no. H3570; Thermo Fisher Scientific, Inc.), and images of the cells were captured using a BZ-9000 fluorescence microscope (Keyence Corporation).

Statistical analysis. In vitro experiments were performed in duplicate and independently repeated three times. Data are expressed as the mean \pm standard error of the mean. Statistical significance was evaluated using a two-tailed unpaired Student's t-test for comparisons between two groups, or one-way analysis of variance with Tukey-Kramer post hoc tests for comparisons among multiple groups. $\mathrm{P}<0.05$ was considered to indicate a statistically significant difference.

\section{Results}

Colchicine treatment attenuates fibrosis in UUO kidneys. No significant renal or liver dysfunction was observed in the UUO-operated animals, as indicated by measurements of serum creatinine and alanine aminotransferase, respectively (Table II). In the UUO kidneys, fibrosis was observed mainly in the interstitium surrounding atrophic or dilated tubules (Fig. 1A). Masson's trichrome staining demonstrated that colchicine treatment significantly suppressed renal interstitial fibrosis compared with the vehicle treatment (Fig. 1A). The number of interstitial cells expressing vimentin, a mesenchymal marker, was significantly 
Table II. Physical and biochemical characteristics of mice on day 14 following UUO.

\begin{tabular}{lccr}
\hline Group & UUO+Vehicle $(\mathrm{n}=8)$ & UUO+Colchicine $(\mathrm{n}=14)$ & P-value \\
\hline Body weight $(\mathrm{g})$ & $26.0 \pm 0.6$ & $25.2 \pm 0.5$ & 0.32 \\
Serum ALT $(\mathrm{IU} / \mathrm{l})$ & $20.1 \pm 0.7$ & $21.6 \pm 3.0$ & 0.70 \\
Serum Cr $(\mu \mathrm{mol} / \mathrm{l})$ & $11.9 \pm 0.6$ & $12.4 \pm 0.5$ & 0.60 \\
\hline
\end{tabular}

Results are presented as the mean \pm standard error of the mean. UUO, unilateral ureteral obstruction; ALT, alanine aminotransferase; Cr, creatinine.
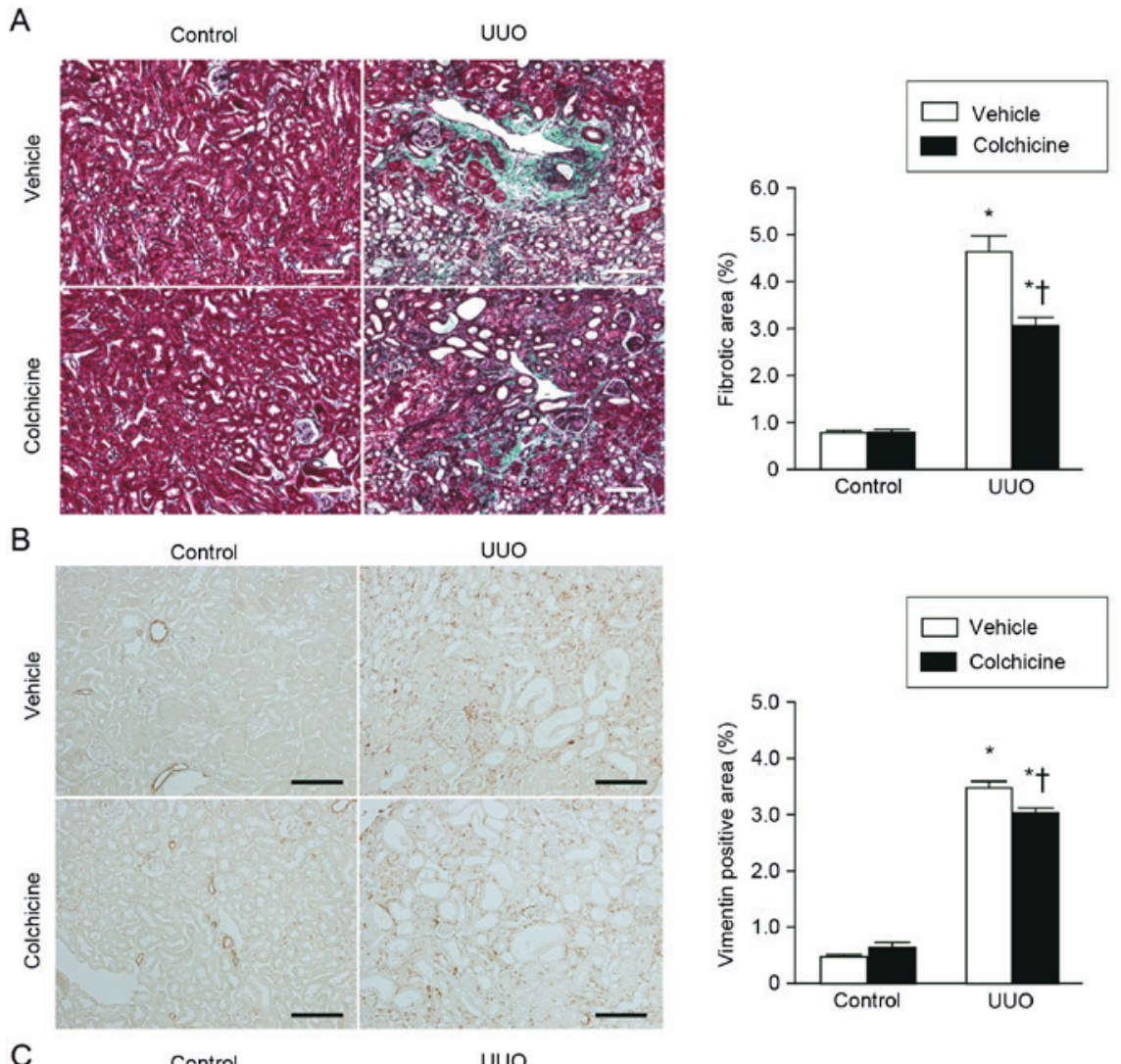

C
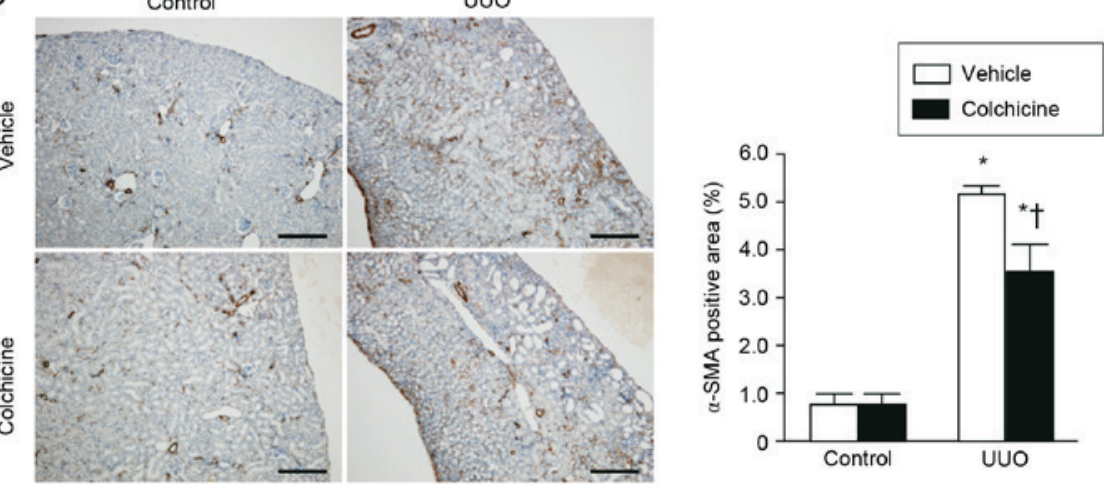

Figure 1. Effects of colchicine on mouse UUO-induced renal fibrosis. Kidney tissue sections from UUO kidneys and control kidneys, treated with vehicle control or colchicine, were evaluated for markers of renal fibrosis. (A) Masson's trichrome staining (scale bar, $100 \mu \mathrm{m}$ ). (B) Vimentin immunohistochemical staining (scale bar, $100 \mu \mathrm{m}$ ). (C) $\alpha$-SMA immunohistochemical staining (scale bar, $200 \mu \mathrm{m}$ ). Quantification of staining is presented as the mean \pm standard error of the mean. ${ }^{*} \mathrm{P}<0.05$ vs. control; ${ }^{\dagger} \mathrm{P}<0.05$ vs. vehicle-treated UUO kidneys. UUO, unilateral ureteral obstruction; $\alpha$-SMA, $\alpha$-smooth muscle actin.

increased in the UUO kidneys compared with the control kidneys; however, this effect was attenuated by colchicine treatment (Fig. 1B). Expression of the myofibroblast marker $\alpha$-SMA was also significantly increased in the UUO kidneys compared with the control kidneys, and this UUO-mediated increase was also attenuated by colchicine treatment (Fig. 1C).

The effects of colchicine treatment on the mRNA expression levels of profibrotic genes fibronectin and $\alpha 1$ type I 

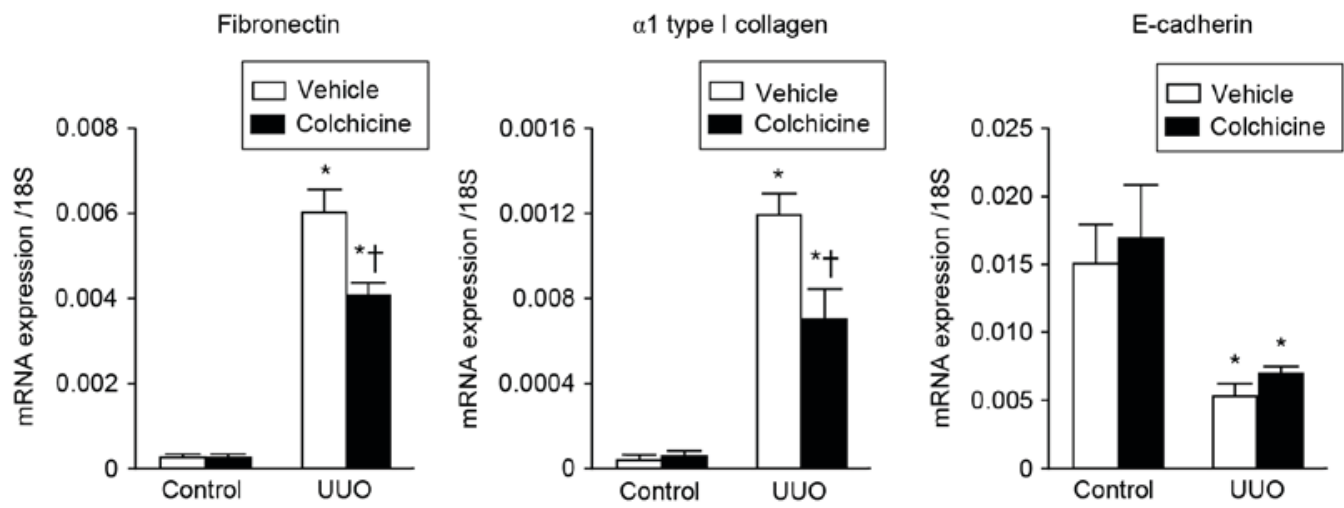

Figure 2. Effects of colchicine on UUO-induced fibrogenic gene expression. mRNA expression levels of fibronectin, $\alpha 1$ type I collagen and E-cadherin were determined in UUO kidney and control kidney tissues by reverse transcription-quantitative polymerase chain reaction. Data were normalized to the $18 \mathrm{~S}$ ribosomal RNA internal reference and expressed as the mean \pm standard error of the mean. ${ }^{*} \mathrm{P}<0.05$ vs. control; ${ }^{\dagger} \mathrm{P}<0.05$ vs. vehicle-treated UUO kidneys. UUO, unilateral ureteral obstruction.

collagen were examined in the UUO and control kidneys. The results demonstrated that the expression levels of fibronectin and $\alpha 1$ type I collagen were higher in the UUO kidneys compared with the control kidneys (Fig. 2). However, expression of these genes in the UUO kidneys was significantly attenuated by colchicine treatment compared with the vehicle treatment (Fig. 2). In addition, the mRNA expression levels of the epithelial adhesion molecule E-cadherin were decreased in the UUO kidneys compared with the control kidneys; however, its expression was not affected by colchicine treatment (Fig. 2).

Colchicine treatment reduces RhoA activity in NRK- $49 \mathrm{~F}$ cells. The effects of colchicine on normal fibroblast activities were further examined in vitro, by measuring the cell viability and RhoA activity of NRK-49F normal rat kidney fibroblast cells. Angiotensin II, colchicine, or co-stimulation with both, had no significant effects on the viability (Fig. 3A) or death (Fig. 3B) of NRK-49F cells. RhoA activity in NRK-49F cells was significantly increased by angiotensin II stimulation (Fig. 3C); however, colchicine treatment significantly suppressed this angiotensin-II-mediated RhoA activity in a dose-dependent manner (Fig. 3C).

Colchicine treatment suppresses angiotensin II-induced NRK-49F cell migration. Cells were stimulated with angiotensin II in the presence or absence of colchicine and migration was examined in vitro $20 \mathrm{~h}$ after wounding. Colchicine treatment inhibited the NRK-49F cell migration in a concentration-dependent manner (Fig. 4A and B). When cells were stained with fluorescent phalloidin for visualization of actin fibers, formation of filopodia was upregulated in the NRK-49F cells treated with angiotensin II, whereas colchicine treatment suppressed this effect (Fig. 4C).

\section{Discussion}

The present study investigated the therapeutic potential of colchicine in UUO-induced renal fibrosis and demonstrated that colchicine treatment ameliorated renal fibrosis and fibrogenic gene expression in mouse UUO kidneys. In addition, in in vitro experiments colchicine treatment inhibited angiotensin II-induced RhoA activation and reduced fibroblast migration. These findings indicated that colchicine may provide a protective effect against kidney fibrosis by suppressing migration of renal fibroblasts.

Mechanisms of existing drugs are continuously being investigated at a molecular level, and identification of novel uses for existing drugs (drug repositioning) has gained popularity (5). Colchicine is a microtubule-depolymerizing drug that has been used to treat gout-associated arthritis (6), Behçet's syndrome (24), primary biliary cirrhosis (25) and familial Mediterranean fever (26). In the present study, colchicine pretreatment was effective for the prevention of renal fibrosis in mice, suggesting that colchicine may be used in the future as a repositioned drug for the treatment of kidney diseases. In a clinical situation however, given the well-known toxicity of colchicine, it is unlikely that colchicine administration will be considered preventively. Therefore, further studies are required to investigate whether colchicine treatment is also effective for the treatment of renal fibrosis following UUO.

Clinically, appropriate blood concentrations for treatment with colchicine range between 0.5 and $3.0 \mathrm{ng} / \mathrm{ml}$ (27). The therapeutic range is narrow, and poisoning is dose-dependent. Gastrointestinal symptoms, including diarrhea, nausea or abdominal pain, are frequently observed in patients, and liver dysfunction or bone marrow suppression are less frequent but severe side effects. In the present study, liver dysfunction and renal damage were not observed in the mice following colchicine administration. The colchicine concentrations used in the in vitro experiments were based on the safe blood concentrations, and the LDH assay revealed that the drug was not cytotoxic in NRK-49F normal renal fibroblasts at these concentrations. The present findings suggested that the beneficial effects of colchicine could be expected even when used at low doses.

In the present study, colchicine was demonstrated to inhibit fibroblast migration in vitro without affecting cell viability, and the inhibitory effect observed was postulated to be due to the suppression of RhoA activity. RhoA is a small GTPase that regulates the formation of stress fibers through Rho kinase (28). Stress fibers create cellular tension and induce rear retraction in cells, a necessary step of cell migration (29). Inhibition of stress fiber formation therefore abolishes the migratory capacity of cells. Both inhibition or activation of 
A

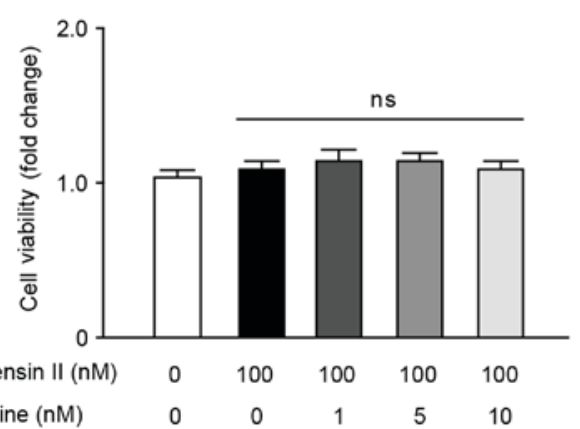

B

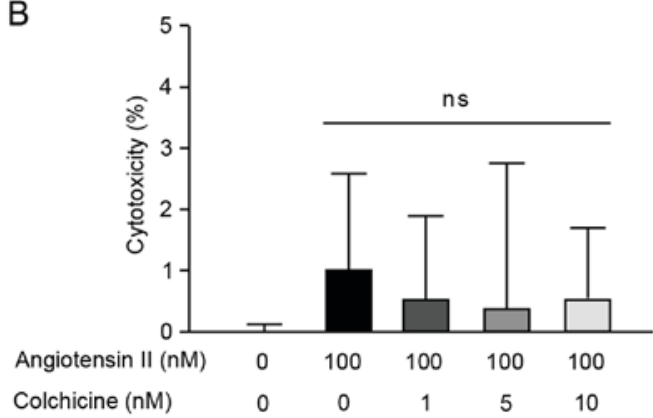

C

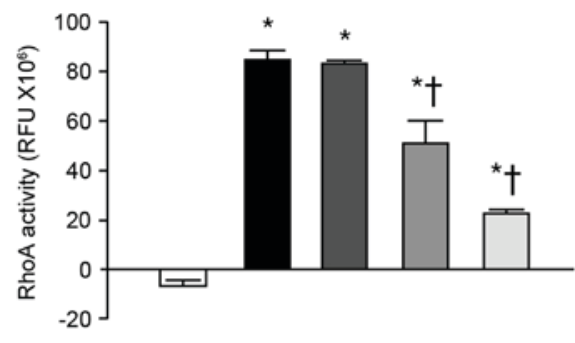

Angiotensin II (nM) $\quad 0 \quad 100 \quad 100 \quad 100 \quad 100$

Colchicine $(\mathrm{nM})$

Figure 3. Effects of colchicine on NRK-49F cells. NRK-49F cells were stimulated with angiotensin II and simultaneously treated with $0,1,5$ or $10 \mathrm{nM}$ colchicine. (A) Cell viability was examined by WST-1 assay. Data represent the fold change over control cells (without angiotensin II and colchicine). (B) Cell toxicity was examined by lactate dehydrogenase assay. (C) RhoA activity. Data are presented as the mean + standard error of the mean $(n=3) .{ }^{*} \mathrm{P}<0.05$ vs. control untreated cells; ${ }^{\dagger} \mathrm{P}<0.05$ vs. $100 \mathrm{nM}$ angiotensinII-treatedcells.WST-1,(2-(4-iodophenyl)-3-(4-nitrophenyl)-5-(2,4disulfophenyl)-2H-tetrazolium sodium salt; RFU, relative fluorescence units; ns, not significant.

Rho decrease cell migration (30), indicating that dynamic Rho regulation is important for cell migration. An association between Rho/Rho-kinase signaling and renal fibrosis has previously been reported (31), and colchicine has been demonstrated to attenuate renal fibrosis via RhoA inhibition in a 5/6 nephrectomized rat model of hypertensive CKD (14). In the present study, the inhibitory effects of colchicine on RhoA/Rho-kinase activation observed in renal fibroblasts further confirmed that this signaling pathway may be important for attenuation of renal fibrosis.

Interstitial recruitment of myofibroblasts, which are responsible for the excessive production and deposition of extracellular matrix during renal fibrosis, occurs at an early stage in UUO kidneys (32). Activated fibroblasts or myofibroblasts have diverse origins, including residential fibroblasts, vascular pericytes, bone marrow cells, and

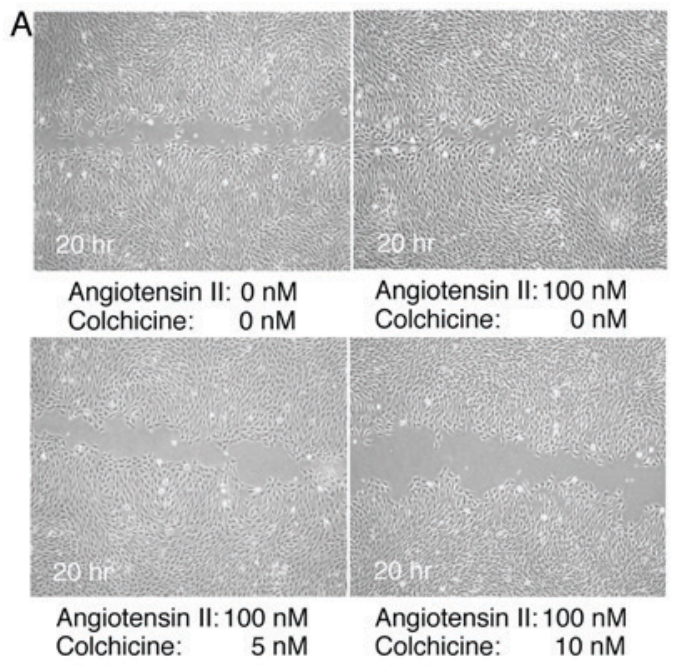

B
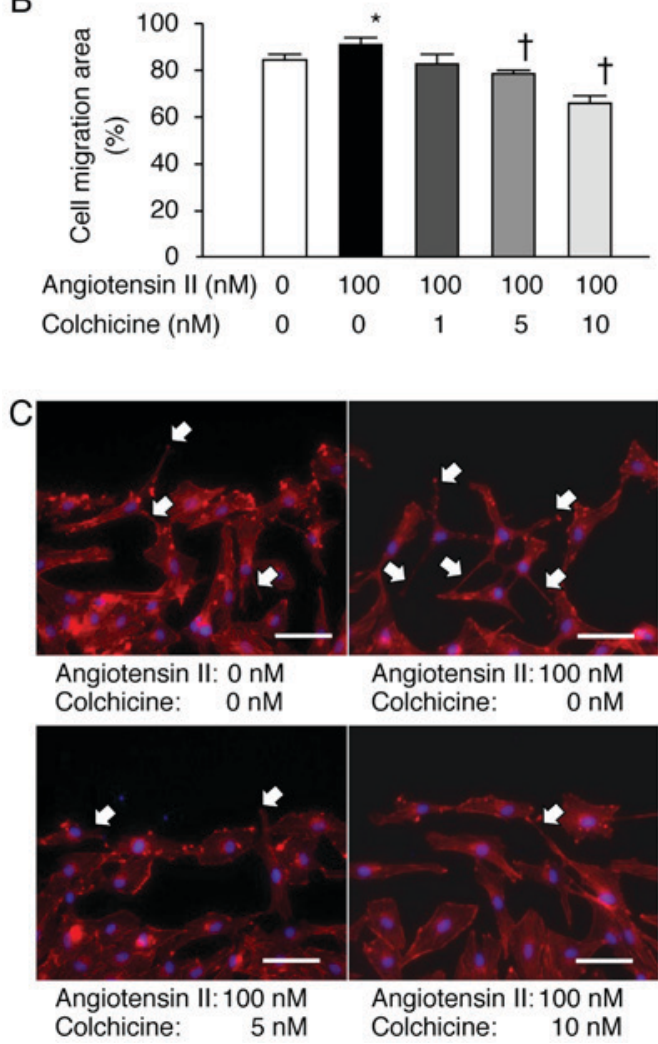

Figure 4. Effects of colchicine on angiotensin II-induced NRK-49F cell migration. (A) In vitro cell migration of NRK-49F fibroblasts was evaluated by the wound-healing assay. Cell migration was assessed at $20 \mathrm{~h}$ post-wounding the cell monolayer. Scale bar $=200 \mu \mathrm{m}$ (B) Quantification of the cell migration area (reflecting the rate by which the cells migrate to fill the scratched area) is expressed as the mean + standard error of the mean $(n=3)$. ${ }^{*} \mathrm{P}<0.05$ vs. untreated control cells; ${ }^{\dagger} \mathrm{P}<0.05$ vs. $100 \mathrm{nM}$ angiotensin II-treated cells. (C) Actin filaments in NRK-49F migrating cells were stained with phalloidin-tetramethylrhodamine isothiocyanate (red), and the nuclei were stained with Hoechst 33342 (blue). The white arrows indicate the formation of filopodia in fibroblasts. Scale bar, $50 \mu \mathrm{m}$.

epithelial-mesenchymal transition (EMT) (33). Loss of E-cadherin expression is one of the markers of EMT (34). In the present study, E-cadherin mRNA expression was decreased in UUO kidneys compared with in control kidneys; however, colchicine treatment did not reverse this 
effect. Therefore, these results indicated that colchicine may have no effect on EMT following UUO.

Although the UUO model is an established renal fibrosis model and suitable for testing the antifibrotic activity of novel compounds, the efficacy of colchicine in other renal fibrosis models needs to be investigated further. In addition, a limitation of the present study is that it did not assess whether colchicine may attenuate fibrosis following UUO (as it was administered in the mice as a pretreatment); therefore, it remains unclear whether colchicine treatment may be effective against advanced fibrosis. Further studies are required to evaluate the efficacy of colchicine in other systems and the effects of colchicine administration following UUO surgery. Although several aspects require further investigation, the present study provides valuable information regarding the effects of colchicine therapy on CKD progression toward fibrosis. The recent results may be used as the basis for developing more effective therapies against renal fibrosis in the future.

\section{Acknowledgements}

The present study was supported in part by a Research Project Grant from Kawasaki Medical School (grant no. 27 Dai-7) and by scholarship donations from Pfizer Academic Contributions, Astellas Research Support and Chugai Pharmaceutical. The authors would like to thank Ms. Etsuko Yorimasa and Ms. Tomoko Taira (Kawasaki Medical School) for animal care and Ms. Satomi Hanada, Ms. Keiko Satoh and Ms. Yoshiko Shirakiya (Kawasaki Medical School) for help with in vitro experiments. This manuscript has been edited and corrected by an experienced proofreader who is a native speaker of English and who is under direct supervision of Honyaku Center, Inc. (Osaka, Japan).

\section{References}

1. Liu Y: Cellular and molecular mechanisms of renal fibrosis. Nat Rev Nephrol 7: 684-696, 2011.

2. Zeisberg M and Neilson EG: Mechanisms of tubulointerstitial fibrosis. J Am Soc Nephrol 21: 1819-1834, 2010.

3. Boor P, Sebekova K, Ostendorf T and Floege J: Treatment targets in renal fibrosis. Nephrol Dial Transplant 22: 3391-3407, 2007.

4. Ramos AM, Gonzalez-Guerrero C, Sanz A, Sanchez-Niño MD, Rodríguez-Osorio L, Martín-Cleary C, Fernández-Fernández B, Ruiz-Ortega $\mathrm{M}$ and Ortiz A: Designing drugs that combat kidney damage. Expert Opin Drug Discov 10: 541-556, 2015.

5. Ashburn TT and Thor KB: Drug repositioning: Identifying and developing new uses for existing drugs. Nat Rev Drug Discov 3: 673-683, 2004.

6. Nuki G: Colchicine: Its mechanism of action and efficacy in crystal-induced inflammation. Curr Rheumatol Rep 10: 218-227, 2008

7. Hastie SB: Interactions of colchicine with tubulin. Pharmacol Ther 51: 377-401, 1991.

8. Slobodnick A, Shah B, Pillinger MH and Krasnokutsky S: Colchicine: Old and new. Am J Med 128: 461-470, 2015.

9. Rodríguez L, Cerbón-Ambriz J and Muñoz ML: Effects of colchicine and colchiceine in a biochemical model of liver injury and fibrosis. Arch Med Res 29: 109-116, 1998.

10. Ledwozyw A: The effect of colchicine and vinblastine on bleomycin-induced lung fibrosis in rats. Acta Physiol Hung 82: 383-389, 1994

11. Disel U, Paydas S, Dogan A, Gulfiliz G and Yavuz S: Effect of colchicine on cyclosporine nephrotoxicity, reduction of TGF-beta overexpression, apoptosis, and oxidative damage: An experimental animal study. Transplant Proc 36: 1372-1376, 2004
12. McClurkin C Jr, Phan SH, Hsu CH, Patel SR, Spicker JK, Kshirsagar AM, Yuan WY and Wiggins RC: Moderate protection of renal function and reduction of fibrosis by colchicine in a model of anti-GBM disease in the rabbit. J Am Soc Nephrol 1: 257-265, 1990.

13. Li JJ, Lee SH, Kim DK, Jin R, Jung DS, Kwak SJ, Kim SH, Han SH, Lee JE, Moon SJ, et al: Colchicine attenuates inflammatory cell infiltration and extracellular matrix accumulation in diabetic nephropathy. Am J Physiol Renal Physiol 297: F200-F209, 2009.

14. Guan T, Gao B, Chen G, Chen X, Janssen M, Uttarwar L, Ingram AJ and Krepinsky JC: Colchicine attenuates renal injury in a model of hypertensive chronic kidney disease. Am J Physiol Renal Physiol 305: F1466-F1476, 2013.

15. Klahr S and Pukerson ML: The pathophysiology of obstructive nephropathy: The role of vasoactive compounds in the hemodynamic and structural abnormalities of the obstructed kidney. Am J Kidney Dis 23: 219-223, 1994.

16. Diamond JR, Kees-Folts D, Ding G, Frye JE and Restrepo NC: Macrophages, monocyte chemoattractant peptide-1 and TGF-beta 1 in experimental hydronephrosis. Am J Physiol 266: F926-F933, 1994.

17. Kaneto H, Morrissey $\mathrm{J}$ and Klahr S: Increased expression of TGF-beta $1 \mathrm{mRNA}$ in the obstructed kidney of rats with unilateral ureteral ligation. Kidney Int 44: 313-321, 1993.

18. Satoh M, Kashihara N, Yamasaki Y, Maruyama K, Okamoto K, Maeshima Y, Sugiyama H, Sugaya T, Murakami K and Makino H: Renal interstitial fibrosis is reduced in angiotensin II type 1a receptor-deficient mice. J Am Soc Nephrol 12: 317-325, 2001.

19. Du F, Li S, Wang T, Zhang HY, Li DT, Du ZX and Wang HQ: Implication of Bcl-2-associated athanogene 3 in fibroblast growth factor-2-mediated epithelial-mesenchymal transition in renal epithelial cells. Exp Biol Med (Maywood) 240: 566-575, 2015.

20. Satoh M, Fujimoto S, Horike H, Ozeki M, Nagasu H, Tomita N Sasaki T and Kashihara N: Mitochondrial damage-induced impairment of angiogenesis in the aging rat kidney. Lab Invest 91: 190-202, 2011.

21. Clark K, Karsch-Mizrachi I, Lipman DJ, Ostell J and Sayers EW: GenBank. Nucleic Acids Res 44: D67-D72, 2016.

22. Dhanasekaran S, Doherty TM and Kenneth J; TB Trials Study Group: Comparison of different standards for real-time PCR-based absolute quantification. J Immunol Methods 354: 34-39, 2010.

23. Bolick DT, Whetzel AM, Skaflen M, Deem TL, Lee J and Hedrick CC: Absence of the G protein-coupled receptor G2A in mice promotes monocyte/endothelial interactions in aorta. Circ Res 100: 572-580, 2007.

24. Yurdakul S, Mat C, Tüzün Y, Ozyazgan Y, Hamuryudan V, Uysal O, Senocak M and Yazici H: A double-blind trial of colchicine in Behçet's syndrome. Arthritis Rheum 44: 2686-2692, 2001.

25. Kaplan MM and Gershwin ME: Primary biliary cirrhosis. N Engl J Med 353: 1261-1273, 2005.

26. La Regina M, Ben-Chetrit E, Gasparyan AY, Livneh A, Ozdogan $\mathrm{H}$ and Manna R: Current trends in colchicine treatment in familial Mediterranean fever. Clin Exp Rheumatol 31 (3 Suppl 77): S41-S46, 2013.

27. Deftereos S, Giannopoulos G, Papoutsidakis N, Panagopoulou V, Kossyvakis C, Raisakis K, Cleman MW and Stefanadis C: Colchicine and the heart: Pushing the envelope. J Am Coll Cardiol 62: 1817-1825, 2013.

28. Murali A and Rajalingam K: Small Rho GTPases in the control of cell shape and mobility. Cell Mol Life Sci 71: 1703-1721, 2014.

29. Pellegrin S and Mellor H: Actin stress fibres. J Cell Sci 120 3491-3499, 2007.

30. Allen WE, Zicha D, Ridley AJ and Jones GE: A role for Cdc42 in macrophage chemotaxis. J Cell Biol 141: 1147-1157, 1998.

31. Takeda Y, Nishikimi T, Akimoto K, Matsuoka $\mathrm{H}$ and Ishimitsu T: Beneficial effects of a combination of Rho-kinase inhibitor and ACE inhibitor on tubulointerstitial fibrosis induced by unilateral ureteral obstruction. Hypertens Res 33: 965-973, 2010.

32. Picard N, Baum O, Vogetseder A, Kaissling B and Le Hir M: Origin of renal myofibroblasts in the model of unilateral ureter obstruction in the rat. Histochem Cell Biol 130: 141-155, 2008.

33. Mack M and Yanagita M: Origin of myofibroblasts and cellular events triggering fibrosis. Kidney Int 87: 297-307, 2015.

34. Zeisberg M and Kalluri R: The role of epithelial-to-mesenchymal transition in renal fibrosis. J Mol Med (Berl) 82: 175-181, 2004. 